\title{
Elevated expression of neuropilin-2 associated with unfavorable prognosis in hepatocellular carcinoma
}

\author{
This article was published in the following Dove Press journal: \\ OncoTargets and Therapy \\ 31 July 2017 \\ Number of times this article has been viewed
}

\begin{abstract}
Xiaogang Dong, ${ }^{1-5, *}$
Wenjia Guo, $6, *$ Shizhen

Zhang, ${ }^{7, *}$ Tianchun Wu, ${ }^{1,3-5}$

Zhongquan Sun, ${ }^{1,3-5}$ Sheng

Yan, 1,3-5 Shusen Zheng',3-5

'Division of Hepatobiliary and Pancreatic Surgery, Department of Surgery, First Affiliated Hospital, School of Medicine,

Zhejiang University, Hangzhou, Zhejiang Province, ${ }^{2}$ Department of Hepatobiliary and Pancreatic Surgery, Affiliated Tumor Hospital of Xinjiang Medical University, Urumqi, Xinjiang Uygur Autonomous Region, ${ }^{3}$ Key Laboratory of Combined MultiOrgan Transplantation, Ministry of Public Health, ${ }^{4}$ Key Laboratory of Organ Transplantation, First Affiliated Hospital, School of Medicine, Zhejiang University, Hangzhou, Zhejiang Province, ${ }^{5}$ Collaborative Innovation Center for Diagnosis Treatment of Infectious Diseases, Hangzhou, Zhejiang Province, ${ }^{6}$ Department of Cancer Research Institute, Affiliated Tumor Hospital of Xinjiang Medical University, Urumqi, Xinjiang Uygur Autonomous Region, ${ }^{7}$ Department of Surgical Oncology, Second Affiliated Hospital, Zhejiang University School of Medicine, Hangzhou, Zhejiang Province, People's Republic of China

*These authors contributed equally to this work
\end{abstract}

Correspondence: Shusen Zheng; Sheng Yan

Division of Hepatobiliary and Pancreatic Surgery, Department of Surgery, First Affiliated Hospital, School of Medicine, Zhejiang University, 79 Qingchun Road, Hangzhou 310003, People's Republic of China

Tel $+8657187236601 ;+8657187236688$

Fax +86 57I $87236884 ;+8657 \mid 87236765$

Email shusenzheng@zju.edu.cn;

shengyan@zju.edu.cn

\begin{abstract}
Neuropilin-2 (NRP2) is a single-pass transmembrane glycoprotein and has recently been detected in several human cancer cells. However, its clinical relevance in hepatocellular carcinoma (HCC) remains unclear. This study aimed at evaluating NRP2 expression and clinicopathological significance in HCC patients. Tissue microarray of $190 \mathrm{HCC}$ patients from the First Affiliated Hospital of Zhejiang University was established, and immunohistochemical staining was performed for NRP2. The Kaplan-Meier analysis and Cox proportional hazard model were used to analyze the survival rate. We found that NRP2 expression in HCC was significantly associated with tumor histological degree $(P=0.023)$ and cirrhosis $(P=0.040)$. Furthermore, NRP2-positive HCC patients demonstrated shorter disease-free survival (DFS) and overall survival (OS) than those of NRP2-negative patients. Then, the multivariate Cox analysis showed that hazard ratios of NRP2-positive patients with DFS and OS were 2.167 (95\% CI: 1.626, 2.889) and 2.317 (95\% CI: 1.548, 3.469), respectively. Our results suggested that NRP2 expression was considered as an independent factor for the prediction of unfavorable prognosis in HCC patients, and we believe that NRP2 could serve as a biomarker of poor prognosis and a novel target in treating HCC tumors.
\end{abstract}

Keywords: NRP2, hepatocellular carcinoma, unfavorable prognosis, immunohistochemistry, disease-free survival, overall survival

\section{Introduction}

Hepatocellular carcinoma (HCC) is one of the most common primary malignancy of the liver and is the second most frequent cause of cancer mortality worldwide. ${ }^{1}$ Approximately 750,000 new HCC cases are diagnosed annually, and half of them are in People's Republic of China. ${ }^{2}$ The prognosis of HCC remains poor due to its high intrahepatic recurrence rate postcurative hepatectomy. ${ }^{3}$ Hence, novel biomarkers that improve the diagnosis and treatment of HCC patients are awaiting discovery.

Neuropilins (NRPs) are highly conserved multifunctional transmembrane glycoproteins. To date, two NRP homologues have been identified in vertebrates, Neuropilin-1 (NRP1) and Neuropilin-2 (NRP2), and are composed of 923 and 926 amino acids, respectively. ${ }^{4}$ NRPs play a regulatory role in nervous system development, as they possess neuronal guidance functions by interacting with plexins for specific secreted members of the semaphoring family..$^{5-7}$ Then, accumulating evidences suggest that NRPs act as multifunctional correctors due to their ability to bind to a wider variety of protein families, including transforming growth factor- $\beta 1$ (TGF- $\beta 1$ ), vascular endothelial growth factor (VEGF), epidermal growth factor, platelet-derived growth factor, and other growth factors, exerting diverse biological functions, such as angiogenesis, immune system regulation, and tumor growth and progression. ${ }^{8-11}$ The two NRP homologues share $44 \%$ amino acid sequence identity and have similar overall 
structure, but their expression patterns, regulation, and ligand binding specificities are significantly different. ${ }^{12}$ NRP1 plays an important role in angiogenesis and vasculogenesis. ${ }^{13}$ Unlike NRP1, NRP2 mainly involves in the formation of lymphatic vessels. ${ }^{14} \mathrm{NRP} 1$ is expressed in the epithelial cells, and some tumors of epithelial origin are likely to express NRP1. NRP1 expression has been detected in several tumor biopsies, such as prostate, mammary, bladder, kidney, colon, pancreas, skin, ovarian, and lung carcinomas. ${ }^{15-20}$ Although NRP2 is not expressed in epithelial cells, its expression is predominant in human melanoma cells, human glioblastoma cells, and human neuroblastoma cells. ${ }^{21}$ However, recent reports also confirmed the expression of NRP2 in lung cancer, prostate cancer, and gastrointestinal cancer. ${ }^{19,22,23}$ In a recent study, Elpek reviewed the role of NRPs in liver diseases and concluded that they were involved in liver regeneration, liver fibrosis, and malignant transformation. ${ }^{24} \mathrm{NRP} 1$ expression was increased in human $\mathrm{HCC}$, and $\sim 50 \%$ of primary HCC samples were positively stained for NRP1. ${ }^{25}$ NRP1 expression in $\mathrm{HCC}$ has been associated with intrahepatic metastasis, TNM classification and portal vein invasion, shorter recurrence-free survival (RFS), and shorter overall survival (OS). ${ }^{26} \mathrm{NRP} 2$ is crucially involved in the elevation of the migratory capability of de-differentiated HCC cells through TGF- $\beta$-dependent pathway. ${ }^{27}$ However, the expression patterns and prognostic values of NRP 2 in HCC patients are not completely understood. Therefore, in this study, we evaluated the NRP2 expression by immunohistochemical staining in HCC samples and found that the NRP2 expression was associated with advanced histological grade tumor and cirrhosis. In addition, NRP2-positive HCC patients had shorter disease-free survival (DFS) and OS than those of NRP2-negative patients. Therefore, we believe that NRP2 could serve as a biomarker of poor prognosis and a novel target in treating HCC patients.

\section{Materials and methods Patients}

A total of 190 paraffin-embedded HCC and matched adjacent normal liver tissue specimens were collected from the First Affiliated Hospital, School of Medicine, Zhejiang University, between July 2010 and August 2012. Inclusion criteria were as follows: 1) if pathological diagnosis confirmed HCC without other primary cancers; 2) if follow-up information was available; and 3) if informed consent was obtained. All the patients showed the absence of lymph node or distant metastasis and received no chemotherapy, radiotherapy, or immunotherapy before the operation.
The available clinical data, such as gender, age, tumor size, hepatitis B virus surface antigen, serum AFP, and TNM classification, were collected for further analyses. The patients were followed up until August 2015. OS and RFS were defined as the interval between surgery and death or recurrence. If recurrence was not diagnosed, patients were censored on the date of death or the last follow-up assessment. The Ethics Committee of the First Affiliated Hospital, School of Medicine, Zhejiang University, approved the use of tissue samples in this study.

\section{Construction of tissue microarray (TMA)}

Tissue cores with a diameter of $0.6 \mathrm{~mm}$ were isolated from paraffin-embedded formalin-fixed tissue and transferred into a recipient paraffin block by using the Beecher Manual Tissue Arrayer (Beecher Instruments, Inc., Sun Prairie, WI, USA). The selected tissue cores were chosen by an experienced pathologist from our hospital based on H\&E staining of tissue sections. Then, consecutive sections of 4-5 $\mu \mathrm{m}$ were cut from TMA blocks and placed on glass slides for subsequent immunohistochemical analysis.

\section{NRP2 immunohistochemistry (IHC)}

The expression of NRP 2 of HCC tissues and matched adjacent normal liver tissues were examined by IHC. Paraffin sections of 4-5 $\mu \mathrm{m}$ were deparaffinized, and antigen was retrieved by boiling in $0.1 \mathrm{M}$ citrate buffer ( $\mathrm{pH}$ 6.0) for 20 minutes. Endogenous peroxidase was inactivated with $0.3 \% \mathrm{H}_{2} \mathrm{O}_{2}$ for 10 minutes. Then, the primary antibody NRP2 (ab185710; dilution 1:100; Abcam, Cambridge, UK) was incubated for overnight at $4^{\circ} \mathrm{C}$. PBS was used as a negative control. Subsequently, the sections were incubated with a biotinylated goat anti-rabbit secondary antibody for 30 minutes at a room temperature. Next, 3,3-diaminobenzidine was applied for 5 and 10 minutes. Each slide was counterstained with hematoxylin for redyeing. Then, the slides were dehydrated, making transparent and sealed.

\section{Scoring of NRP2 expression}

Two independent observers evaluated the degree of NRP2 immunostaining. The results were scored according to the intensity and percentage of NRP2-positive cells. Staining intensity was scored as follows: absent staining (negative, 0), weak staining (1), moderate staining (2), and strong staining (3). The percent of positive cells was grouped into 4 categories, in which 1 was given for $0 \%-10 \%, 2$ for $11 \%-50 \%, 3$ for $51 \%-80 \%$, and 4 for $81 \%-100 \%$. The two scores were then multiplied to calculate the final score. NRP2 
expression was considered low if the final score was $\leq 3$ and otherwise high. ${ }^{26}$

\section{Statistical analysis}

SPSS version 21.0 software (IBM SPSS, Armonk, NY, USA) was used for data analysis. Pearson's $\chi^{2}$ test was used to analyze the association between NRP2 and clinicopathological factors. OS and DFS curves were plotted by using the Kaplan-Meier method and compared by using log-rank test. Univariate analysis was performed with the log-rank test. The prognostic significance of NRP2 expression and all clinical covariates were measured by using Cox regression analysis. $P<0.05$ was set as statistically significant difference.

\section{Results}

\section{Characteristics of the eligible HCC patients}

We included 190 eligible HCC patients who were undergoing curative resection in the First Affiliated Hospital, School of Medicine, Zhejiang University, between July 2010 and August 2012. All the patients involved had no preoperative lymph node and distant metastasis. The patient's age range was from 23 to 89 years at the time of surgery. Of the 190 patients who were included in the study, 178 patients were male, and 12 were female. All the patients were followed up via telephone interviews, and the follow-up time ranged between 6 and 61 months.

\section{NRP2 expression correlates with histological grade tumor and cirrhosis in HCC patients}

NRP2 was expressed in human HCC cell lines and plays an important role in tumor cell migration. ${ }^{27}$ In order to further explore regarding the expression of NRP2 in carcinogenesis of HCC, we analyzed the expression of NRP2 in $190 \mathrm{HCC}$ tissues on a TMA by immunohistochemistry. Table 1 summarizes the clinical characteristics of the patients. NRP2 expression was mainly observed in cytoplasm and cytomembrane (Figure 1). A total of 55.3\% (105 of 190) of HCC patients demonstrated NRP2-positive staining. As shown in Table 1, NRP2 expression was significantly associated with higher tumor grading $(P=0.023)$. NRP2 expression was observed in $55.7 \%$ of HCC patients with Grade II and $61.5 \%$ with Grade III tumors, whereas only $27.8 \%$ of well-differentiated HCC patients (Grade I) demonstrated NRP2-positive staining. In addition, we found that the expression rate of NRP2 was higher in HCC patients without cirrhosis ( $P=0.003$ ); $38.9 \%$ of HCC patients without cirrhosis demonstrated NRP2-positive
Table I NRP2 protein expression and pathoclinical characteristics of HCC patients

\begin{tabular}{|c|c|c|c|}
\hline \multirow[t]{2}{*}{ Characteristics } & \multicolumn{2}{|l|}{$n=190$} & \multirow[t]{2}{*}{$P$-valu } \\
\hline & $\begin{array}{l}\text { Number } \\
\text { of patients }\end{array}$ & $\begin{array}{l}\text { Number of NRP2- } \\
\text { positive (\%) }\end{array}$ & \\
\hline \multicolumn{3}{|l|}{ Age (years) } & \multirow[t]{3}{*}{0.619} \\
\hline$\leq 50$ & 48 & $25(52.1)$ & \\
\hline$>50$ & 142 & $80(56.3)$ & \\
\hline \multicolumn{3}{|l|}{ Gender } & \multirow[t]{3}{*}{0.770} \\
\hline Male & 178 & $99(55.6)$ & \\
\hline Female & 12 & $6(50.0)$ & \\
\hline \multicolumn{3}{|l|}{$\mathrm{HBV}$ antigen } & \multirow[t]{3}{*}{1.000} \\
\hline$(-)$ & 38 & $21(55.3)$ & \\
\hline$(+)$ & 152 & $84(55.3)$ & \\
\hline \multicolumn{3}{|l|}{$\operatorname{AFP}(n g / m L)$} & \multirow[t]{3}{*}{0.293} \\
\hline$\geq 20$ & 72 & $36(50.0)$ & \\
\hline$<20$ & 118 & $69(58.5)$ & \\
\hline \multicolumn{3}{|l|}{ Histological grade } & \multirow[t]{4}{*}{$0.023^{*}$} \\
\hline 1 & 18 & $5(27.8)$ & \\
\hline II & 120 & $68(55.7)$ & \\
\hline III & 52 & $32(6 \mid .5)$ & \\
\hline \multicolumn{3}{|l|}{ Tumor size } & \multirow[t]{4}{*}{0.462} \\
\hline TI & 154 & $83(53.9)$ & \\
\hline $\mathrm{T} 2$ & 14 & $8(57.14)$ & \\
\hline $\mathrm{T} 3$ & 22 & $14(63.6)$ & \\
\hline \multicolumn{3}{|l|}{ AJCC stage } & \multirow[t]{4}{*}{0.450} \\
\hline 1 & 156 & $84(53.8)$ & \\
\hline ॥ & 14 & $8(57.14)$ & \\
\hline III & 20 & II (55.0) & \\
\hline \multicolumn{3}{|l|}{ Tumor thrombosis } & \multirow[t]{3}{*}{0.496} \\
\hline Negative & 168 & 91 (54.2) & \\
\hline Positive & 22 & $14(63.2)$ & \\
\hline \multicolumn{3}{|c|}{ Microvascular invasion } & \multirow[t]{3}{*}{0.377} \\
\hline Negative & 114 & $66(57.9)$ & \\
\hline Positive & 76 & $39(51.3)$ & \\
\hline \multicolumn{3}{|l|}{ Cirrhosis } & \multirow[t]{3}{*}{$0.040^{*}$} \\
\hline Negative & 36 & 14 (38.9) & \\
\hline Positive & 154 & 91 (6I.I) & \\
\hline
\end{tabular}

Note: $* P<0.05$ was considered statistically significant.

Abbreviations: AJCC, American Joint Committee on Cancer; HBV, hepatitis B virus; HCC, hepatocellular carcinoma; NRP2, neuropilin-2.

staining, whereas $61.1 \%$ of $\mathrm{HCC}$ with cirrhosis showed NRP2 expression. Then, we used nonconditional logistic analysis to examine the correlations of NRP2 expression with these clinical parameters (Table 2). The results indicated that NRP2 expression showed statistically significant impact on the risk of advanced histological grade tumor and cirrhosis. The adjusted odds ratio (OR) of NRP2-positive HCC with advanced histological grade tumor was 1.431 (95\% CI: $1.025,2.414)$, whereas the adjusted OR of NRP2-positive HCC with cirrhosis was 0.306 (95\% CI: 0.142, 0.657). All these results implicated that NRP2 expression was associated with $\mathrm{HCC}$ with a de-differentiated phenotype and without cirrhosis. 

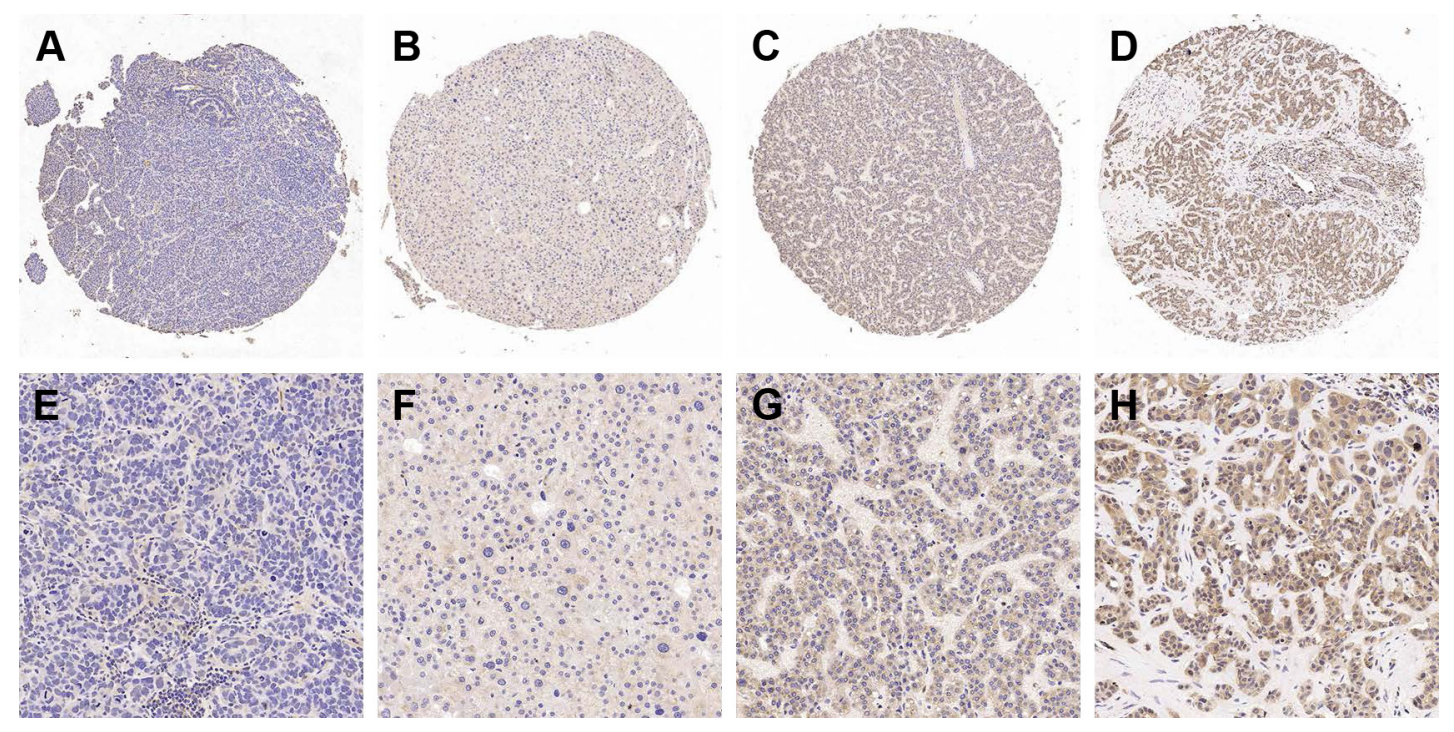

Figure I Immunohistochemical staining of NRP2 expression in HCC tissues with (A) negative, (B) weakly positive, (C) moderately positive, and (D) strongly positive in low magnification (40x); and (E) negative, (F) weakly positive, $(\mathbf{G})$ moderately positive, and (H) strongly positive in high magnification (200×) under a microscope (Olympus, Tokyo, Japan).

Abbreviations: HCC, hepatocellular carcinoma; NRP2, neuropilin-2.

\section{NRP2 expression correlates with poor prognosis in HCC patients}

In order to better understand the prognostic value of NRP2 in HCC patients, Kaplan-Meier survival analysis based on NRP2 immunostaining of primary tumors was used to

Table 2 Nonconditional logistic analysis for NRP2 and some clinicopathological characteristics

\begin{tabular}{|c|c|c|}
\hline Variables & OR (95\% Cl) & Adjusted OR $(95 \% \mathrm{Cl})$ \\
\hline \multicolumn{3}{|c|}{ Histological grade } \\
\hline I-II & Reference & Reference \\
\hline III & $1.516(1.010,2.275)^{*}$ & I.43I $(1.025,2.414)^{*}$ \\
\hline \multicolumn{3}{|l|}{ HBV antigen } \\
\hline Negative & Reference & Reference \\
\hline Positive & $0.862(0.493,1.507)$ & $0.946(0.509,1.759)$ \\
\hline \multicolumn{3}{|l|}{ Tumor size } \\
\hline TI-T2 & Reference & Reference \\
\hline T3 & $0.940(0.679,1.302)$ & $1.005(0.647,1.559)$ \\
\hline \multicolumn{3}{|l|}{ AFP (ng/mL) } \\
\hline$\geq 20$ & Reference & Reference \\
\hline$<20$ & I.408 (0.829, 2.39I) & $1.606(0.863,2.990)$ \\
\hline \multicolumn{3}{|c|}{ Tumor thrombosis } \\
\hline Negative & Reference & Reference \\
\hline Positive & $1.067(0.537,2.123)$ & $1.006(0.379,2.673)$ \\
\hline \multicolumn{3}{|c|}{ Microvascular invasion } \\
\hline Negative & Reference & Reference \\
\hline Positive & $0.630(0.399,1.004)$ & $0.619(0.373,1.028)$ \\
\hline \multicolumn{3}{|l|}{ Cirrhosis } \\
\hline Negative & Reference & Reference \\
\hline Positive & $0.352(0.176,0.704)^{*}$ & $0.306(0.142,0.657)^{*}$ \\
\hline
\end{tabular}

Notes: Adjusted OR, adjusted by age and tumor location in multivariate logistic analysis; $* P<0.05$ was considered statistically significant.

Abbreviations: HBV, hepatitis B virus; NRP2, neuropilin-2; OR, odds ratio. evaluate the relationship between NRP2 protein staining and OS/DFS in 190 HCC samples. As shown in Figure 2, NRP2 expression was significantly associated with shorter DFS and OS. The median survival time of NRP2-positive HCC patients was 29 months, which was much shorter than that of NRP2-negative HCC patients (37 months). The median DFS time of NRP2-positive HCC patients was 12 months, whereas the median DFS time of NRP2-negative HCC patients was 18 months. In order to determinate the independent prognostic role of NRP2 in HCC patients, univariate and multivariate Cox analyses were performed, and Table 3 presents the results. The factors such as age (hazard ratio $[\mathrm{HR}]=1.022,95 \% \mathrm{CI}: 1.006,1.038$ ), AFP level (HR: $1.634,95 \%$ CI: 1.049, 2.554), histological grade (HR: 1.524, 95\% CI: 1.135, 2.046), American Joint Committee on Cancer stage (HR: 2.225, 95\% CI: 1.966, 2.554), tumor thrombosis (HR: $2.744,95 \%$ CI: 1.784, 4.221), microvascular invasion (HR: 3.837, 95\% CI: 2.680, 5.493), and cirrhosis (HR: 1.686, $95 \%$ CI: 1.001, 2.846) were independent risk factors associated with OS according to univariate Cox analysis, whereas the factors such as age, AFP level, and histological grade were not associated with poor OS according to multivariate analysis (Table 3). Multivariate HR of NRP2-positive with OS was 2.317 (95\% CI: 1.548, 3.469), indicating that NRP2positive was an independent predictive factor of poor OS in HCC patients. Multivariate analysis showed that gender (HR: 0.431, 95\% CI: 0.235, 0.790), tumor thrombosis (HR: 2.480 , 95\% CI: 1.538, 4.001), microvascular invasion (HR: 2.430, 95\% CI: 1.834, 3.219), cirrhosis (HR: 2.312, 95\% CI: 1.544 , 
A

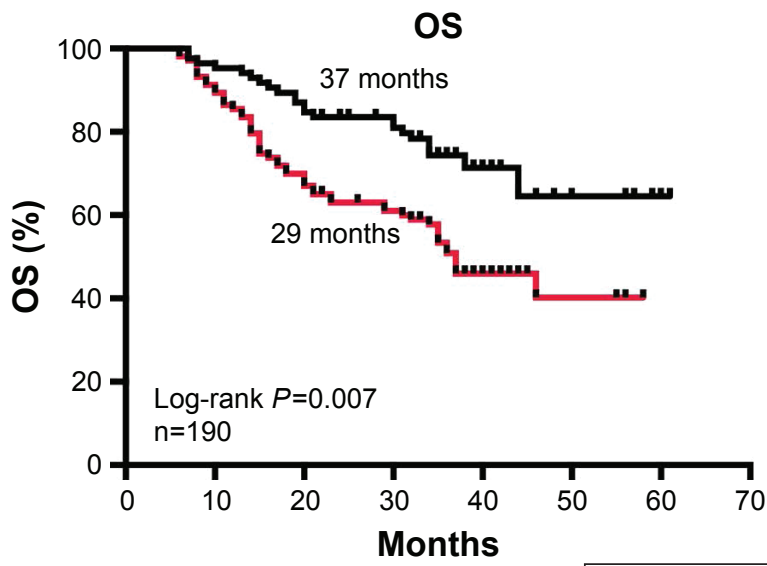

Months

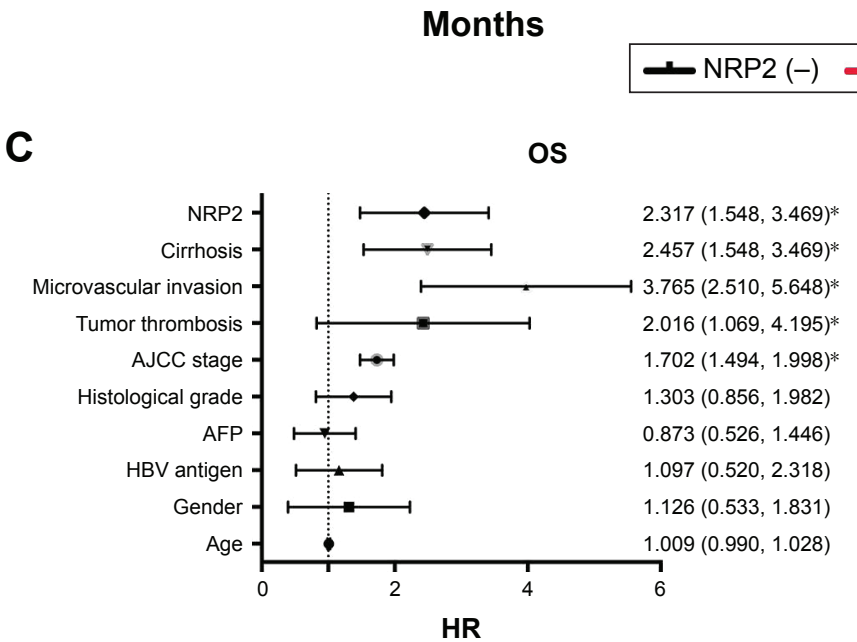

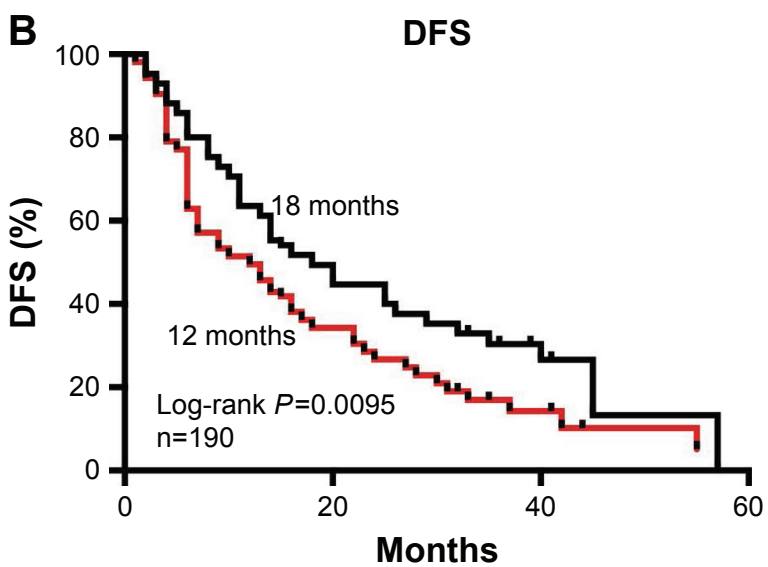

Months

D

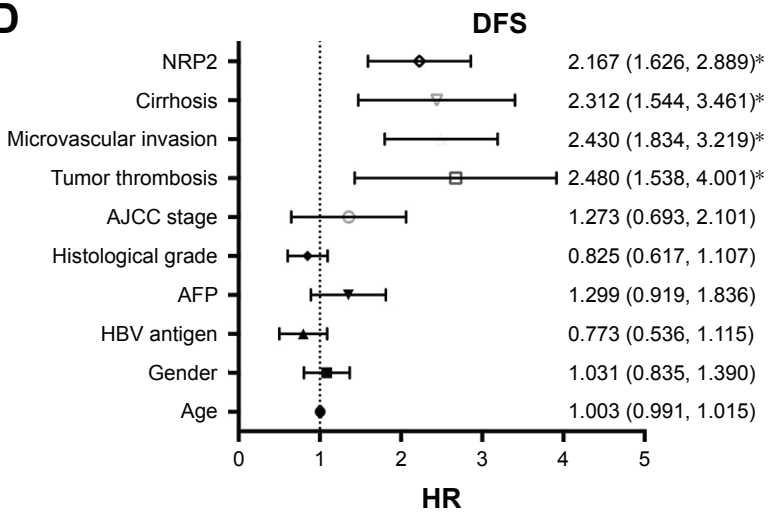

Figure 2 Prognostic significance of NRP2 expression in HCC patients.

Notes: (A) Kaplan-Meier analysis of NRP2 expression for HCC with OS; (B) DFS analysis of NRP2 expression for HCC; (C) forest plots of the results of multivariate Cox analysis for OS; (D) forest plots of the DFS results of multivariate Cox analysis. $* P<0.05$.

Abbreviations: AJCC, American Joint Committee on Cancer; DFS, disease-free survival; HBV, hepatitis B virus; HCC, hepatocellular carcinoma; HR, hazard ratio; NRP2, neuropilin-2; OS, overall survival.

3.461), and NRP2 expression (HR: 2.167, 95\% CI: 1.626, 2.889) were independent risk factors for shorter DFS. All the above data indicated that NRP2 expression demonstrated shorter DFS and OS for HCC patients.

\section{Discussion}

Several studies have investigated regarding NRP1 and deduced the expression of NRP1 in many cancer types and their association with tumor-related angiogenesis and

Table 3 Univariate and multivariate Cox analysis for NRP2 and survival of HCCs

\begin{tabular}{|c|c|c|c|c|}
\hline \multirow[t]{3}{*}{ Variables } & \multicolumn{2}{|l|}{ OS } & \multicolumn{2}{|l|}{ DFS } \\
\hline & Univariate & Multivariate & Univariate & Multivariate \\
\hline & HR $(95 \% \mathrm{Cl})$ & HR (95\% Cl) & HR (95\% Cl) & HR (95\% Cl) \\
\hline Age ( $>50$ years vs $\leq 50$ years) & $1.022(1.006,1.038)^{*}$ & $1.009(0.990,1.028)$ & $1.006(0.996,1.017)$ & 1.003 (0.99I, I.015) \\
\hline Gender (male vs female) & $0.986(0.482,2.015)$ & $1.097(0.520,2.318)$ & I.I $36(0.899,1.529)$ & I.03। $(0.835,1.390)$ \\
\hline HBV antigen (+ vs -) & $1.385(0.629,1.550)$ & I.I26 (0.533, I.83I) & $0.818(0.533,1.168)$ & $0.773(0.536,1.115)$ \\
\hline $\operatorname{AFP}(\geq 20 \mathrm{ng} / \mathrm{mL}$ vs $<20 \mathrm{ng} / \mathrm{mL})$ & $1.634(1.049,2.554)^{*}$ & $0.873(0.526, I .446)$ & $1.418(1.056,1.904)^{*}$ & $1.299(0.919,1.836)$ \\
\hline Histological grade (III vs I/II) & $1.524(1.135,2.046)^{*}$ & $1.303(0.856,1.982)$ & I.I75 (0.952, I.45I) & $0.825(0.617,1.107)$ \\
\hline AJCC stage (III vs I/II) & $2.225(1.966,2.554)^{*}$ & $1.702(1.494,1.998)^{*}$ & $1.306(1.082,1.577)^{*}$ & $1.273(0.693,2.101)$ \\
\hline Tumor thrombosis (+ vs - ) & $2.744(I .784,4.22 I)^{*}$ & $2.016(1.069,4.195)^{*}$ & $2.837(1.980,4.065)^{*}$ & $2.480(\mathrm{I} .538,4.00 \mathrm{I})^{*}$ \\
\hline Microvascular invasion (+ vs -) & $3.837(2.680,5.493)^{*}$ & $3.765(2.510,5.648)^{*}$ & $2.384(1.856,3.063)^{*}$ & $2.430(1.834,3.219)^{*}$ \\
\hline Cirrhosis (+ vs -) & $1.686(1.00 \mathrm{I}, 2.846)^{*}$ & $2.457(1.548,3.469)^{*}$ & $1.622(1.143,2.302)^{*}$ & $2.312(1.544,3.46 I)^{*}$ \\
\hline NRP2 (+ vs -) & $1.969(1.371,2.826)^{*}$ & $2.317(1.548,3.469)^{*}$ & $1.530(1.188,1.970)^{*}$ & $2.167(1.626,2.889)^{*}$ \\
\hline
\end{tabular}

Note: $* P<0.05$ was considered statistically significant.

Abbreviations: AJCC, American Joint Committee on Cancer; DFS, disease-free survival; HBV, hepatitis B virus; HCC, hepatocellular carcinoma; HR, hazard ratio; NRP2, neuropilin-2; OS, overall survival. 
tumor progression. ${ }^{28-31}$ Previous studies have demonstrated the upregulation of NRP1 in HCC, contributing to tumor growth and increasing time to recurrence..$^{25,32,33}$ However, less information is known regarding the expression of NRP2 in malignant tumors. Emerging evidences have demonstrated that NRP2 was expressed in non-small cell lung cancer patients, ${ }^{34}$ osteosarcoma, ${ }^{35}$ colorectal cancer, ${ }^{36}$ and other types of cancers. ${ }^{10}$ In lung cancer cells, NRP2 enhanced cellular migration, invasion, and tumor sphere formation in response to TGF $\beta$ signaling, and NRP2 expression was correlated with metastatic disease and poor prognosis. ${ }^{34}$ In our study, we found that more than half of HCC patients demonstrated NRP2 expression, and the lower the differentiated degree of the tumor, the higher the expression rate of NRP2. Survival analysis indicated that NRP2 expression in HCC was significantly associated with poor prognosis, shorter DFS, and shorter OS. In addition, our study also suggested that the tumor thrombosis and microvascular invasion were poor prognostic factors ${ }^{37-39}$ and independent factors associated with OS and DFS in HCC.

Consistent with the previous study results, ${ }^{27}$ we showed that NRP2 expression was significantly correlated with less differentiation in HCC patients. The hedgehog (Hh) signaling system has been implicated in the regulation of cellular differentiation, proliferation, tissue polarity, stem cell population, and carcinogenesis. ${ }^{40-42} \mathrm{Hh}$ signaling plays an important role in maintaining the undifferentiated phenotype in cancer cells, whereas NRP2 is a positive regulator of Hh signal transduction. ${ }^{43}$ Therefore, we speculate that NRP2 expression in HCC regulates Hh signaling in the de-differentiated state of tumor cells.

Angiogenesis is a vital process in tumor progression, and VEGF family and their tyrosine kinase receptors play pivotal roles in the physiological and pathological angiogenesis. ${ }^{44}$ Initially, NRP2 was identified as a semaphorin receptor in the mediation of axon guidance. ${ }^{33}$ Now, it has been proposed that NRP2 functions to enhance VEGF receptor signaling and the modulation of lymphatic endothelial cell migration. ${ }^{45} \mathrm{NRP} 2$ interacts with VEGF-C and VEGF-D, inducing lymphovascular development and stimulating lymph node metastasis via VEGFR-3. ${ }^{46,47}$ NRP2 also interacts with the N-terminal domain of hepatocyte growth factor (HGF), pleiotropic cytokine, and potent proangiogenic molecule, enhancing the HGF-mediated angiogenesis. ${ }^{48}$ In addition, blocking of NRP2 leads to the inhibition of tumor lymphangiogenesis and reduction in metastasis of the primary tumor. ${ }^{47}$ Consistently, our results indicated that NRP2 expression in HCC allows tumor to metastasize more easily and showed a shorter DFS.
Furthermore, angiogenesis was closely associated with liver fibrosis and has reported that hepatic stellate cells secreted NRP1 to induce angiogenesis in liver fibrosis. ${ }^{49}$ Our study showed that $61.1 \%$ of HCC patients with cirrhosis showed NRP2-positive, whereas only $38.9 \%$ patients showed NRP2-negative.

\section{Conclusion}

To sum, the present study demonstrated that NRP2 expression in HCC patients was positively associated with pathological differentiation and cirrhosis. In addition, NRP2 expression was also significantly associated with the progression of tumor malignancy and poorer survival rates in patients with HCC. These results suggested that NRP2 was recognized as a novel biomarker for prognosis and a potential therapeutic target for the treatment of HCC.

\section{Acknowledgments}

This study was supported by Joint Funds of the Natural Science Foundation of the Xinjiang Uygur Autonomous Region (No 2016D01C372).

\section{Disclosure}

The authors report no conflicts of interest in this work.

\section{References}

1. Chen W, Zheng R, Baade PD, et al. Cancer statistics in China, 2015. CA Cancer J Clin. 2016;66(2):115-132.

2. Liu L, Liu Y, Liu J, et al. Genetic variants in pseudogene E2F3P1 confer risk for HBV-related hepatocellular carcinoma in a Chinese population. J Biomed Res. 2013;27(3):215-219.

3. Fong ZV, Tanabe KK. The clinical management of hepatocellular carcinoma in the United States, Europe, and Asia: a comprehensive and evidence-based comparison and review. Cancer. 2014;120(18): 2824-2838.

4. Narayanan N, Su N, Bedard P. Inhibitory and stimulatory effects of fluoride on the calcium pump of cardiac sarcoplasmic reticulum. Biochim Biophys Acta. 1991;1070(1):83-91.

5. Giger RJ, Cloutier JF, Sahay A, et al. Neuropilin-2 is required in vivo for selective axon guidance responses to secreted semaphorins. Neuron. 2000;25(1):29-41.

6. He Z, Tessier-Lavigne M. Neuropilin is a receptor for the axonal chemorepellent Semaphorin III. Cell. 1997;90(4):739-751.

7. Fujisawa H. Discovery of semaphorin receptors, neuropilin and plexin, and their functions in neural development. J Neurobiol. 2004;59(1): 24-33.

8. Staton CA, Kumar I, Reed MW, Brown NJ. Neuropilins in physiological and pathological angiogenesis. J Pathol. 2007;212(3):237-248.

9. Uniewicz KA, Fernig DG. Neuropilins: a versatile partner of extracellular molecules that regulate development and disease. Front Biosci. 2008; 13:4339-4360.

10. Grandclement C, Borg C. Neuropilins: a new target for cancer therapy. Cancers (Basel). 2011;3(2):1899-1928.

11. Raimondi C, Ruhrberg C. Neuropilin signalling in vessels, neurons and tumours. Semin Cell Dev Biol. 2013;24(3):172-178.

12. Klagsbrun M, Takashima S, Mamluk R. The role of neuropilin in vascular and tumor biology. Adv Exp Med Biol. 2002;515:33-48. 
13. Kitsukawa T, Shimono A, Kawakami A, Kondoh H, Fujisawa H Overexpression of a membrane protein, neuropilin, in chimeric mice causes anomalies in the cardiovascular system, nervous system and limbs. Development. 1995;121(12):4309-4318.

14. Yuan L, Moyon D, Pardanaud L, et al. Abnormal lymphatic vessel development in neuropilin 2 mutant mice. Development. 2002;129(20): 4797-4806.

15. Wey JS, Stoeltzing O, Ellis LM. Vascular endothelial growth factor receptors: expression and function in solid tumors. Clin Adv Hematol Oncol. 2004;2(1):37-45.

16. Neufeld G, Kessler O. The semaphorins: versatile regulators of tumour progression and tumour angiogenesis. Nat Rev Cancer. 2008; 8(8):632-645.

17. Neufeld G, Shraga-Heled N, Lange T, Guttmann-Raviv N, Herzog Y, Kessler O. Semaphorins in cancer. Front Biosci. 2005;10:751-760.

18. Fukahi K, Fukasawa M, Neufeld G, Itakura J, Korc M. Aberrant expression of neuropilin-1 and -2 in human pancreatic cancer cells. Clin Cancer Res. 2004;10(2):581-590.

19. Okon IS, Ding Y, Coughlan KA, et al. Aberrant NRP-1 expression serves as predicator of metastatic endometrial and lung cancers. Oncotarget. 2016;7(7):7970-7978.

20. Luo M, Hou L, Li J, et al. VEGF/NRP-1 axis promotes progression of breast cancer via enhancement of epithelial-mesenchymal transition and activation of NF-kappaB and beta-catenin. Cancer Lett. 2016;373(1):1-11.

21. Bielenberg DR, Pettaway CA, Takashima S, Klagsbrun M. Neuropilins in neoplasms: expression, regulation, and function. Exp Cell Res. 2006;312(5):584-593.

22. Goel HL, Chang C, Pursell B, et al. VEGF/neuropilin-2 regulation of Bmi-1 and consequent repression of IGF-IR define a novel mechanism of aggressive prostate cancer. Cancer Discov. 2012;2(10):906-921.

23. Samuel S, Gaur P, Fan F, et al. Neuropilin-2 mediated beta-catenin signaling and survival in human gastro-intestinal cancer cell lines. PLoS One. 2011;6(10):e23208.

24. Elpek GO. Neuropilins and liver. World J Gastroenterol. 2015;21(23): 7065-7073.

25. Berge M, Allanic D, Bonnin P, et al. Neuropilin-1 is upregulated in hepatocellular carcinoma and contributes to tumour growth and vascular remodelling. J Hepatol. 2011;55(4):866-875.

26. Zhang Y, Liu P, Jiang Y, et al. High expression of neuropilin-1 associates with unfavorable clinicopathological features in hepatocellular carcinoma. Pathol Oncol Res. 2016;22(2):367-375.

27. Wittmann P, Grubinger M, Groger C, et al. Neuropilin-2 induced by transforming growth factor-beta augments migration of hepatocellular carcinoma cells. BMC Cancer. 2015;15:909.

28. Jubb AM, Strickland LA, Liu SD, Mak J, Schmidt M, Koeppen H. Neuropilin-1 expression in cancer and development. J Pathol. 2012; 226(1):50-60.

29. Zachary IC. How neuropilin-1 regulates receptor tyrosine kinase signalling: the knowns and known unknowns. Biochem Soc Trans 2011;39(6):1583-1591.

30. Chaudhary B, Khaled YS, Ammori BJ, Elkord E. Neuropilin 1: function and therapeutic potential in cancer. Cancer Immunol Immunother. 2014;63(2):81-99.

31. Schuch G, Machluf M, Bartsch G Jr, et al. In vivo administration of vascular endothelial growth factor (VEGF) and its antagonist, soluble neuropilin-1, predicts a role of VEGF in the progression of acute myeloid leukemia in vivo. Blood. 2002;100(13):4622-4628.

OncoTargets and Therapy

\section{Publish your work in this journal}

OncoTargets and Therapy is an international, peer-reviewed, open access journal focusing on the pathological basis of all cancers, potential targets for therapy and treatment protocols employed to improve the management of cancer patients. The journal also focuses on the impact of management programs and new therapeutic agents and protocols on
32. Zhuang PY, Wang JD, Tang ZH, et al. Peritumoral Neuropilin-1 and VEGF receptor-2 expression increases time to recurrence in hepatocellular carcinoma patients undergoing curative hepatectomy. Oncotarget. 2014;5(22):11121-11132.

33. Yaqoob U, Cao S, Shergill U, et al. Neuropilin-1 stimulates tumor growth by increasing fibronectin fibril assembly in the tumor microenvironment. Cancer Res. 2012;72(16):4047-4059.

34. Gemmill RM, Nasarre P, Nair-Menon J, et al. The neuropilin 2 isoform NRP2b uniquely supports TGFbeta-mediated progression in lung cancer. Sci Signal. 2017;10(462):pii: eaag0528.

35. Boro A, Arlt MJ, Lengnick H, et al. Prognostic value and in vitro biological relevance of Neuropilin 1 and Neuropilin 2 in osteosarcoma. Am J Transl Res. 2015;7(3):640-653.

36. Staton CA, Koay I, Wu JM, Hoh L, Reed MW, Brown NJ. Neuropilin-1 and neuropilin-2 expression in the adenoma-carcinoma sequence of colorectal cancer. Histopathology. 2013;62(6):908-915.

37. Cho CS, Gonen M, Shia J, et al. A novel prognostic nomogram is more accurate than conventional staging systems for predicting survival after resection of hepatocellular carcinoma. J Am Coll Surg. 2008; 206(2):281-291.

38. Pandey D, Lee KH, Wai CT, Wagholikar G, Tan KC. Long term outcome and prognostic factors for large hepatocellular carcinoma (10 cm or more) after surgical resection. Ann Surg Oncol. 2007;14(10): 2817-2823.

39. Yeh CN, Chen MF, Lee WC, Jeng LB. Prognostic factors of hepatic resection for hepatocellular carcinoma with cirrhosis: univariate and multivariate analysis. J Surg Oncol. 2002;81(4):195-202.

40. Jia Y, Wang Y, Xie J. The Hedgehog pathway: role in cell differentiation, polarity and proliferation. Arch Toxicol. 2015;89(2):179-191.

41. Grzelak CA, Martelotto LG, Sigglekow ND, et al. The intrahepatic signalling niche of hedgehog is defined by primary cilia positive cells during chronic liver injury. J Hepatol. 2014;60(1):143-151.

42. Witek RP, Yang L, Liu R, et al. Liver cell-derived microparticles activate hedgehog signaling and alter gene expression in hepatic endothelial cells. Gastroenterology. 2009;136(1):320-330.e2.

43. Hillman RT, Feng BY, Ni J, et al. Neuropilins are positive regulators of Hedgehog signal transduction. Genes Dev. 2011;25(22): 2333-2346.

44. Djordjevic S, Driscoll PC. Targeting VEGF signalling via the neuropilin co-receptor. Drug Discov Today. 2013;18(9-10):447-455.

45. Favier B, Alam A, Barron P, et al. Neuropilin-2 interacts with VEGFR-2 and VEGFR-3 and promotes human endothelial cell survival and migration. Blood. 2006;108(4):1243-1250.

46. Soker S, Miao HQ, Nomi M, Takashima S, Klagsbrun M. VEGF165 mediates formation of complexes containing VEGFR-2 and neuropilin-1 that enhance VEGF165-receptor binding. J Cell Biochem . 2002;85(2):357-368.

47. Caunt M, Mak J, Liang WC, et al. Blocking neuropilin-2 function inhibits tumor cell metastasis. Cancer Cell. 2008;13(4):331-342.

48. Sulpice E, Plouet J, Berge M, Allanic D, Tobelem G, Merkulova-Rainon T. Neuropilin-1 and neuropilin-2 act as coreceptors, potentiating proangiogenic activity. Blood. 2008;111(4):2036-2045.

49. Taura K, De Minicis S, Seki E, et al. Hepatic stellate cells secrete angiopoietin 1 that induces angiogenesis in liver fibrosis. Gastroenterology. 2008;135(5):1729-1738.

\section{Dovepress}

patient perspectives such as quality of life, adherence and satisfaction. The manuscript management system is completely online and includes a very quick and fair peer-review system, which is all easy to use. Visit http://www.dovepress.com/testimonials.php to read real quotes from published authors. 